provided a rare opportunity to precisely measure the masses of binary stars.

The triple system is about 1,000 parsecs from Earth. The smaller duo have masses of about one-fifth and onequarter that of the Sun, and orbit one another in 1.76 days. They take about 34 days to go around their larger partner, which has a mass 1.3 times that of the Sun. Joshua Carter of the Harvard-Smithsonian Center for Astrophysics in Cambridge, Massachusetts, and his team calculated the masses using the space-based Kepler telescope by observing the dimming of light as the binary stars passed in front of the larger star. They produced a model that fitted the data to determine the dynamics and characteristics of the low-mass pair.

Science doi:10.1126/

science.1201274 (2011)

\section{NEUROSCIENCE}

\section{Ringing in the brain}

People with tinnitus hear a ringing even when there is no sound. The prevailing theory blames a malfunctioning auditory system. However, it seems that abnormalities in the brain's limbic regions, which determine which

$\dot{\bar{s}}$ sensations are important and how they are experienced, may also be involved.

Josef Rauschecker and his colleagues at Georgetown University in Washington DC used functional magnetic resonance imaging to scan the brains of 22 volunteers, half of whom had tinnitus, while they listened to various sounds. Patients with tinnitus showed heightened activity in the nucleus accumbens - a key limbic region - when presented with sounds that matched the frequency of the 'ringing' in their ears. They also had anatomical differences in the ventromedial prefrontal cortex, another limbic area.

The researchers suggest that an abnormal limbic system elevates the perceived importance of the tinnitus sound or fails to suppress it, and that interactions between the auditory and limbic systems may be at the root of this disorder.

Neuron 69, 33-43 (2011)

\section{DRUG DEVELOPMENT}

\section{Promoter predicts drug results}

A clinical trial of a drug to treat behavioural features of the genetic disorder fragile $\mathrm{X}$ syndrome has revealed a genetic signature that seems to predict which patients will respond to the treatment.

Fragile X syndrome results from excessive methylation of a regulatory section - called the promoter - of the FMR1 gene. This silences the gene and increases signalling in the pathway for the brain receptor mGluR5. The drug candidate, AFQ056, blocks mGluR5 activation.

Baltazar Gomez-Mancilla at the Novartis Institutes for Biomedical Research in Basel, Switzerland, and his group assessed the drug's effects on the behaviour of 30 men who had either a fully or partly methylated FMR1 promoter region. Seven with a fully methylated promoter showed behavioural improvements after 19-20 days, whereas the behaviour of those with only partial methylation did not differ significantly between drug and placebo treatments. Sci. Transl. Med. 3, 64ra1 (2011)

\section{THEORETICAL PHYSICS}

\section{Relativity starts your car}

Relativity is generally invoked in lofty thought experiments often involving fast-moving spacecraft, but new work shows that it also applies to the everyday automobile.

Cars are started using lead-acid batteries, which generate energy using electrochemical reactions between lead compounds and sulphuric acid. Rajeev Ahuja of Uppsala University in Sweden and his colleagues modelled

COMMUNITY CHOICE

The most viewed papers in science

PHYLOGENETICS

\title{
How one elephant became two
}

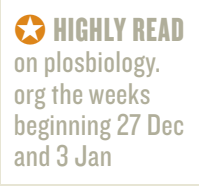

Whether savannah- and forest-dwelling African elephants belong to the same or different species has long been the subject of debate. An analysis of the creatures' genetic ancestry confirms that the two species are separate.

Nadin Rohland at Harvard Medical School in Boston, Massachusetts, and her co-workers sequenced the nuclear genomes of the forest elephant (Loxodonta cyclotis) and the savannah one (L. africana), as well as that of the Asian elephant (Elephas maximus). They also extracted and sequenced DNA from the extinct woolly mammoth (Mammuthus primigenius) and American mastodon (Mammut americanum) — ancient ancestors of today's elephants. By comparing all of these genomes, the team found that the forest and savannah elephants diverged into separate species between 7.1 million and 1.9 million years ago - much earlier than previously proposed. PLoS Biol. 8, e1000564 (2010)

For more on this research, see go.nature.com/91a4zy

the reactions and found that as electrons move at high speed around a lead nucleus, their energy levels change owing to relativity. The authors conclude that the change accounts for $1.7-1.8$ volts of a standard 2.13-volt lead-acid cell.

Phys. Rev. Lett. 106, 018301 (2011)
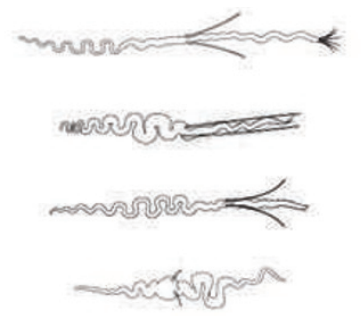

\section{EVOLUTIONARY BIOLOGY}

\section{The many styles of sperm}

Of all the cell types in the animal kingdom, the most diverse are sperm, which can be adorned with tails, hairs, bristles and more. It seems that sex drives the evolution of sperm morphology.

Lukas Schärer at the University of Basel in Switzerland and his colleagues watched 16 species of promiscuous hermaphroditic flatworm (Macrostomum), which have a variety of sperm shapes (pictured), mating under a microscope. After sex, some species suck out the ejaculate, possibly as a way of selecting which sperm are ultimately accepted.

The researchers found that those species that exhibit this sucking behaviour have ornate sperm with features such as a pair of long bristles emerging at the mid-point and a tail resembling a paint brush. These appendages can become lodged in the female orifice after copulation, preventing the sperm from being sucked out. Species that don't remove sperm have evolved simpler sperm that tend to be smaller and lack hairs or bristles.

Proc. Natl Acad. Sci. USA doi:10.1073/pnas.1013892108 (2011)

For a longer story on this research, see go.nature. com/8jvjws

\section{$\rightarrow$ NATURE.COM}

For the latest research published by Naturevisit:

www.nature.com/latestresearch 